\title{
Analisando a dacriocistorrinostomia endoscópica: dificuldades e soluções
}

\author{
Analyzing endoscopic dacryocystorhinostomy:difficulties and solutions
}

Denis Knijnik $^{1}$

\footnotetext{
Trabalho realizado no Hospital Petrópolis - Porto Alegre

${ }^{1}$ Instrutor de Plástica Ocular. Hospital Petrópolis, Porto Alegre (RS) - Brasil.

Endereço para correspondência: Rua Cel. Bordini, 830 - conj. 303 - Porto Alegre (RS) CEP 90450-030

E-mail: kdenis@portoweb.com.br

Recebido para publicação em 09.11.2005

Última versão recebida em 30.11.2006

Aprovação em 11.12.2006

Nota Editorial: Depois de concluída a análise do artigo sob sigilo editorial e com a anuência da $\mathrm{Dr}^{\mathrm{a}}$. Simone H. D. Von Faber Bison e do Dr. Eurípedes da Mota Moura sobre a divulgação de seus nomes como revisores, agradecemos suas participações neste processo.
}

\section{RESUMO}

Objetivo: Verificar a incidência de dificuldades operatórias em dacriocistorrinostomias endoscópicas, assim como propor soluções. Métodos: Estudo retrospectivo que verificou nossos registros de 268 dacriocistorrinostomias endoscópicas endonasais, em 241 pacientes, realizadas de outubro de 1999 a agosto de 2005. A idade dos pacientes variou de 2 a 92 anos (média: 49,1 anos). A uncinectomia (remoção de parte do processo uncinado) foi utilizada como acesso à fossa lacrimal, não havendo necessidade de brocas. Resultados: Houve dificuldades operatórias em 37 $(13,8 \%)$ procedimentos. As dificuldades mais comuns foram: hemorragia $(n=12)$ e anormalidades da anatomia nasal $(n=14)$. Houve ainda dificuldade em alguns casos de mucocele, células etmoidais anteriores e processo uncinado resistente. Das alterações nasais que dificultaram a cirurgia, a mais comum foi desvio de septo, seguida de pólipos, hipertrofia da concha média e fratura da parede lateral. Complicações ocorreram em apenas 5 $(1,9 \%)$ casos, incluindo epistaxe pós-operatória $(n=3)$, enfisema subcutâneo na face e deslocamento do tubo de silicone. Conclusão: A dacriocistorrinostomia endoscópica endonasal com uncinectomia mostrou-se segura, com um mínimo de complicações no pós-operatório. Embora a maioria das cirurgias tenha transcorrido sem dificuldades, este procedimento pode ser tecnicamente difícil, principalmente na presença de hemorragia ou espaço nasal reduzido.

Descritores: Dacriocistorinostomia/métodos; Doenças do aparelho lacrimal; Obstrução dos ductos lacrimais/cirurgia; Endoscopia/métodos; Estudos retrospectivos

\section{INTRODUÇÃ̃O}

A dacriocistorrinostomia endoscópica endonasal é uma técnica eficaz no tratamento das obstruções do ducto nasolacrimal ${ }^{(1)}$. Este acesso endonasal ganhou grande impulso nas duas últimas décadas com a utilização crescente de endoscópios, permitindo melhor visão das estruturas nasais.

Comparada à dacriocistorrinostomia convencional por via externa, a via endonasal apresenta as vantagens de evitar cicatriz, não lesar a veia angular, ser menos traumática e de execução mais rápida ${ }^{(2-3)}$.

Por via endonasal, a abertura óssea para o saco lacrimal é, em geral, iniciada anteriormente à concha média. Outro método de realizar esta osteotomia seria iniciar-se mais posteriormente, com a remoção do processo uncinado (uncinectomia) ${ }^{(4)}$. Esta abordagem geralmente dispensa o uso de brocas, utilizando apenas o saca-bocado ${ }^{(5)}$.

Apesar dos avanços tecnológicos, dificuldades ainda são encontradas ao realizar a dacriocistorrinostomia endoscópica endonasal ${ }^{(6)}$. São estas dificuldades técnicas, além da necessidade de instrumentos específicos, que 
constituem as desvantagens da via endonasal em relação à via externa $^{(1)}$. O relatório da Academia Americana de Oftalmologia sobre a dacriocistorrinostomia endonasal apontou uma curva de aprendizado significativa para o oftalmologista iniciante na técnica ${ }^{(7)}$. É necessário familiarizar-se com as dificuldades e complicações da via endonasal a fim de procurar evitá-las ou lidar adequadamente com elas.

Este estudo tem por objetivo verificar a incidência de dificuldades trans-operatórias em nossa casuística de dacriocistorrinostomias endoscópicas com uncinectomia, assim como apresentar soluções.

\section{MÉTODOS}

Foram revistos os registros de nossas cirurgias no período de outubro de 1999 a agosto de 2005. Os registros mostraram um total de 301 encaminhamentos para a cirurgia por via endonasal, dos quais 33 (11\%) foram contra-indicados devido a achados do exame nasal prévio que dificultariam muito ou impossibilitariam a técnica. O exame foi feito com vasoconstrição tópica da mucosa.

Foram realizadas 268 dacriocistorrinostomias endoscópicas endonasais em 241 pacientes. A idade dos pacientes operados variou de 2 a 92 anos (média: 49,1 anos), tendo sido 179 do sexo feminino e 89 do sexo masculino (feminino/masculino 2,01:1).

A anestesia, na maioria dos adultos, foi local com sedação e as crianças foram operadas sob anestesia geral. Os endoscópios utilizados foram os rígidos de $4 \mathrm{~mm}$ de diâmetro, especialmente os de 0 e 30 graus (Figura 1).

A técnica cirúrgica utilizada foi a dacriocistorrinostomia endoscópica endonasal com uncinectomia. Após vasoconstrição e anestesia da mucosa nasal, a osteotomia iniciou pela penetração, com bisturi em foice, na porção superior do pro-

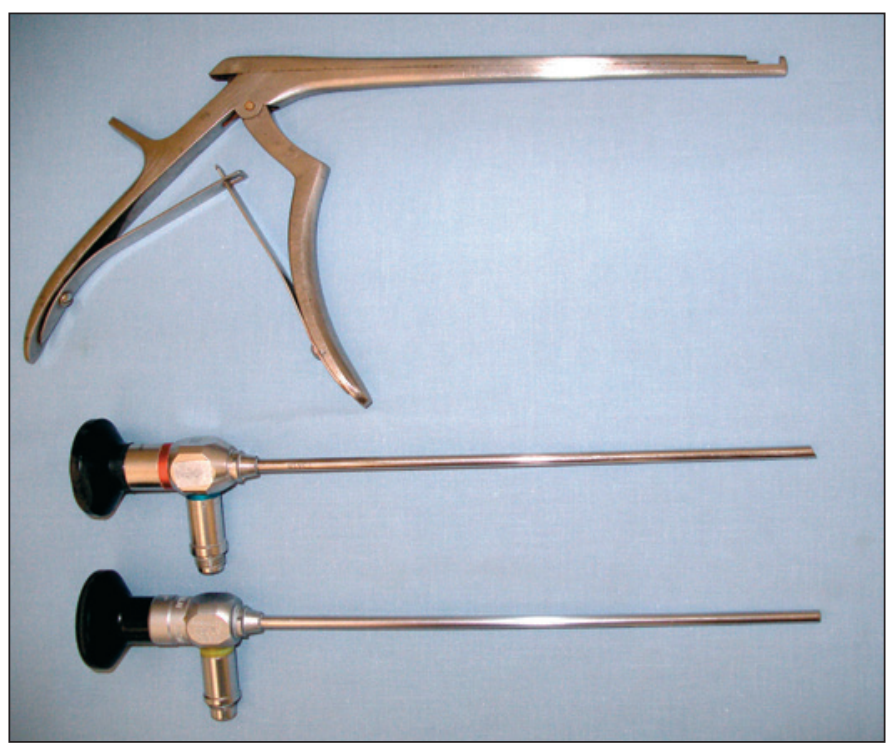

Figura 1 - Endoscópios rígidos de $30^{\circ}$ e $0^{\circ}$ e pinça saca-bocado utilizada para a osteotomia cesso uncinado e sua excisão (uncinectomia). A proximidade do processo uncinado ao saco lacrimal é mostrada na figura 2.

Cauterização e afastamento da mucosa foram opcionais. Quando não deslocada antes da osteotomia, a mucosa foi removida junto com osso pelo saca-bocado de Kerrison. A abertura do saco lacrimal foi facilitada pela indentação de uma sonda introduzida pelo canalículo superior. Parte de sua parede medial foi removida.

Não foi necessário tamponamento nasal na maioria dos casos e os pacientes não ficaram hospitalizados. Houve entubação das vias lacrimais com silicone em 18 casos.

\section{RESULTADOS}

Dentre as 268 dacriocistorrinostomias endoscópicas endonasais realizadas, houve registro de dificuldades trans-operatórias em $37(13,8 \%)$ procedimentos. A tabela 1 apresenta a

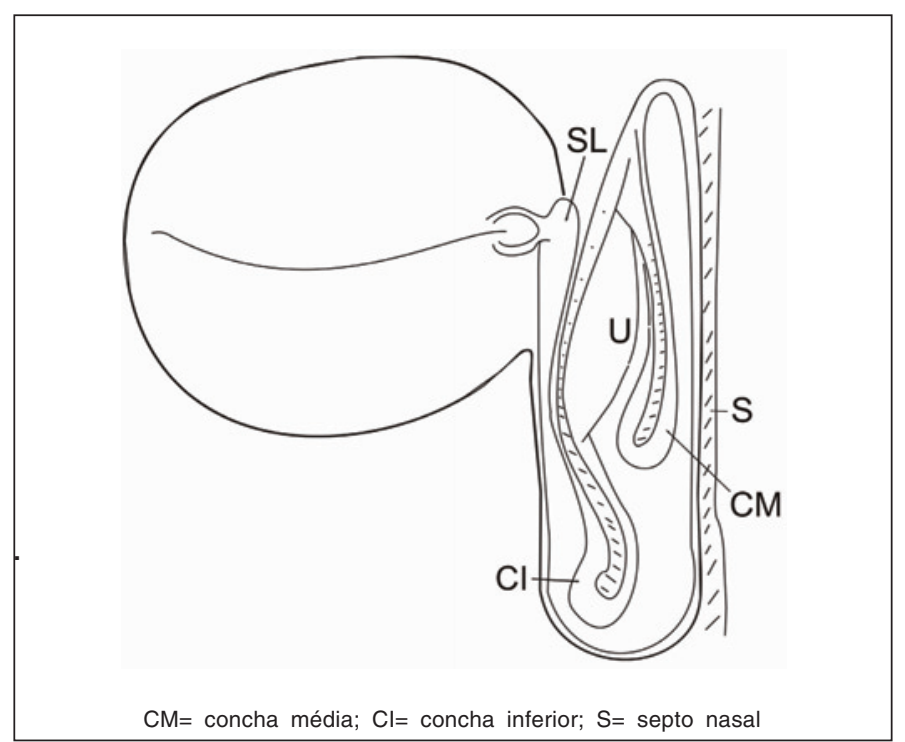

Figura 2 - Ilustração esquemática mostrando a proximidade entre 0 processo uncinado (U) e o saco lacrimal (SL)

\begin{tabular}{|lcc|}
\hline \multicolumn{3}{|c|}{$\begin{array}{c}\text { Tabela 1. Fontes de dificuldade trans-operatória em 268 acriocis- } \\
\text { torrinostomias endoscópicas endonasais }\end{array}$} \\
& $\mathbf{n}$ & $\%$ \\
Hemorragia & 12 & 4,5 \\
Intensa & 7 & 2,6 \\
Moderada & 5 & 1,9 \\
Alterações nasais & 14 & 5,2 \\
Desvio de septo & 7 & 2,6 \\
Fossa nasal estreita & 4 & 1,5 \\
Pólipos & 1 & 0,4 \\
Hipertrofia corneto médio & 1 & 0,4 \\
Fratura & 1 & 0,4 \\
Mucocele & 5 & 1,9 \\
Células etmoidais & 3 & 1,1 \\
Proc. uncinado resistente & 3 & 1,1 \\
\hline
\end{tabular}


incidência de cirurgias em que foram encontradas dificuldades e suas causas. As dificuldades cirúrgicas mais comuns foram anormalidades anatômicas nasais e hemorragia. Além delas, houve dificuldade transoperatória devido à presença de mucocele do saco lacrimal, células etmoidais muito anteriores ou hipertróficas e processo uncinado muito resistente. As alterações encontradas na anatomia endonasal foram classificadas em desvio de septo, fossa nasal estreita não especificada, pólipos nasais, hipertrofia da concha média e fratura da parede lateral da fossa nasal. Os pólipos nasais e a hipertrofia da concha foram ressecados nas cirurgias.

Complicações pós-operatórias ocorreram em apenas $5(1,9 \%)$ casos. Elas incluíram 3 casos de epistaxe pós-operatória $(1,1 \%), 1$ enfisema subcutâneo da face e 1 deslocamento do tubo de silicone. Nenhuma epistaxe requereu transfusão. Um dos pacientes com epistaxe necessitou ser hospitalizado por uma noite para observação. $\mathrm{O}$ enfisema subcutâneo cedeu espontaneamente nos primeiros dias. Em nenhuma das cirurgias dos casos com complicação houve registro de terem ocorrido dificuldades transoperatórias.

De 301 encaminhamentos para DRC por via endonasal, o exame nasal prévio contra-indicou esta abordagem em 33 casos. As alterações nasais encontradas nestes 33 casos foram: 24 $(72,7 \%)$ casos de desvio de septo importante; $3(9,1 \%)$ casos de aderências ou sinéquias; $2(6,1 \%)$ fossas nasais estreitas não especificadas; $2(6,1 \%)$ casos de pólipos; $2(6,1 \%)$ fraturas. Estes pacientes foram encaminhados para dacriocistorrinostomia por via externa ou para correção cirúrgica das anormalidades.

\section{DISCUSSÃO}

Menção ao acesso endonasal para o saco lacrimal, na literatura médica ocidental, remonta a um artigo de Caldwell ${ }^{(8)}$, de 1893. Entretanto, historicamente, a idéia de abrir o saco lacrimal a partir da cavidade nasal é mais antiga: já é citada em 1465 , em livro do cirurgião turco S. Sabuncuoglu ${ }^{(9)}$.

Nos anos 50 do século XX, ainda sem endoscópios de fibra óptica, Bjork ${ }^{(10)}$ achava útil operar o septo de $1 / 3$ dos pacientes de dacriocistorrinostomia endonasal para facilitar a operação.

É provável que, sem endoscopia, pequenos desvios do septo fossem suficientes para perturbar a visualização, a passagem e a movimentação dos instrumentos. Entretanto, um artigo atual de oftalmologista ${ }^{(1)}$ que pratica a via endonasal sem endoscopia não faz referência a dificuldades com o septo nasal. Seus casos de desvio de septo, porém, são detectados em exame nasal prévio.

O processo uncinado, visível endoscopicamente como uma saliência no meato médio, constitui uma janela para o saco lacrimal mais posterior do que as outras formas de acesso endonasal. Devido a essa localização posterior, este acesso está mais propenso a obstáculos, não só pelos desvios anteriores do septo, mas também pelos desvios posteriores. A uncinectomia é também sensível a desvios de septo posteriores por necessitar de espaço para afastar o corneto médio.
A prevalência de desvio de septo na população em geral pode atingir mais de $20 \%$ e tende a aumentar com a idade ${ }^{(12)}$. É, portanto, de se esperar que o exame nasal para candidatos a dacriocistorrinostomia detecte um número significativo de obstruções. O exame nasal permite prever dificuldades transoperatórias nos casos de desvio de septo importante ou hipertrofia de corneto médio, mas somente durante o ato cirúrgico saberemos se desvios menos acentuados vão perturbar a execução da técnica. Apesar disso, septoplastia concomitante só ocasionalmente é necessária, provavelmente em menos de $5 \%$ dos $\operatorname{casos}^{(13)}$. Na literatura recente, encontramos trabalhos apresentando necessidade de septoplastia variando de $0,3 \%$ a $30 \%$ nas dacriocistorrinostomias endonasais ${ }^{(7,14-16)}$.

A não ser que haja fortes razões para selecionar a via endonasal para um paciente com desvio de septo importante diagnosticado previamente pelo exame nasal, é sensato optar pela via externa, evitando a necessidade de recorrer a uma septoplastia.

Medidas para reduzir a dificuldade causada por anormalidades nasais incluem vasoconstrição tópica rigorosa, uso de endoscópios e instrumentos mais finos, septoplastia e turbinectomia média. Entretanto, no que se refere à septoplastia concomitante, concordamos com os que consideram que a incisão da mucosa septal aumenta o sangramento e o risco de sinéquias entre ele e a osteotomia ${ }^{(11)}$, preferindo que a cirurgia não seja estendida ao septo nasal.

Em nossa série, o segundo fator dificultando as cirurgias foi hemorragia. Hemorragia transoperatória leve é esperada em grande parte dos pacientes. Mesmo hemorragias pouco intensas podem comprometer a visão. A hemostasia é crítica e diretamente relacionada com medidas pré-operatórias, como evitar o uso prévio de medicamentos anticoagulantes, controle de hipertensão arterial, vasoconstrição adequada e cuidados para não lacerar a mucosa nasal desnecessariamente.

Cauterização da mucosa, uso de aspirador/cautério e sistemas de aspiração/irrigação são úteis ${ }^{(14)}$, assim como tamponamento nasal, infiltração da mucosa com anestésico com adrenalina e colocação de algodões com vasoconstritor no local do sangramento. A colocação de gelfoam também é sugerida ${ }^{(17)}$.

A necessidade de tamponamento pós-operatório em nossa série foi pequena, tendo sido usado especialmente nos três casos citados de complicação por epistaxe pós-operatória.

A penetração de células etmoidais durante a dacriocistorrinostomia é bastante freqüente. A fossa lacrimal está em proximidade direta com as células etmoidais anteriores. Verificouse que a pneumatização etmoidal chega tão anterior a ponto de penetrar no processo nasal da maxila em $40 \%$ das órbitas ${ }^{(18)}$. Encontrando-se células etmoidais ao iniciar a osteotomia, é necessário abrir a célula para alcançar a fossa lacrimal.

Entre os pacientes com epífora, a presença de mucocele é bastante freqüente. Tsirbas ${ }^{(19)}$ encontrou mucocele em um terço dos pacientes operados. Dos casos de mucocele em nossa casuística, em apenas 5 houve dificuldades técnicas. Devido à dificuldade de passagem da sonda ao saco lacrimal, este é aberto sem auxílio da indentação feita pela sonda. 
Em pacientes que tiveram fratura da face, a hiperostose secundária ou a anatomia alterada dos ossos junto ao saco e canal lacrimal podem tornar a dacriocistorrinostomia endonasal tecnicamente difícil ou impossível ${ }^{(7)}$.

A incidência de complicações em nossa série foi baixa (menos de $2 \%$ ), equiparando-se a outros estudos endonasais pré$\operatorname{vios}^{(15,20)}$. Nossa complicação mais comum foi epistaxe pósoperatória, tratada com tamponamento nasal. Outras duas complicações, enfisema subcutâneo e extrusão do tubo de silicone, são também relatadas na literatura associadas a dacriocistorrinostomia endoscópica endonasal ${ }^{(20-21)}$. Outros estudos referem complicações que não tivemos, como gordura orbitária herniada, hemorragia retrobulbar e paresia do reto medial $^{(7)}$.

\section{CONCLUSÃO}

A dacriocistorrinostomia endoscópica endonasal com uncinectomia mostrou ser uma cirurgia segura, tendo havido um mínimo de complicações pós-operatórias. Sua utilidade, porém, apresenta limitações. Não foram todos os pacientes encaminhados que puderam submeter-se à via endonasal, pois o exame endoscópico prévio detectou, em $11 \%$ deles, alterações importantes da anatomia nasal que impossibilitariam a técnica.

A maioria das dacriocistorrinostomias endoscópicas endonasais transcorre sem dificuldades. Entretanto, este procedimento pode ser tecnicamente difícil na presença de hemorragia, espaço nasal reduzido, células etmoidais, dor, processo uncinado resistente e em alguns casos de mucocele.

\section{ABSTRACT}

Purpose: To assess the incidence of operative difficulties in endonasal endoscopic dacryocystorhinostomies, as well as to suggest solutions. Methods: This retrospective study searched for reports of complications and intraoperative difficulties in our records of 268 endoscopic endonasal dacryocystorhinostomies, in 241 patients, performed from October 1999 to August 2005. The patients' age ranged from 2 to 92 years (mean: 49.1 years). Uncinectomy (partial excision of the uncinate process) was employed as the entry to the lacrimal fossa, no drills being needed for the osteotomy. Results: There were operative difficulties in $37(13.8 \%)$ procedures. The most frequent sources of difficulty were hemorrhage $(n=12)$ and nasal anatomy abnormalities $(n=14)$. Less common sources were lacrimal sac mucocele, anterior ethmoid cells and resistant uncinate process. Of the nasal alterations disturbing surgery, the most common was septal deviation, followed by polyps, hypertrophied middle turbinate and fracture of the lateral wall. Complications were observed in $5(1.9 \%)$ cases, including postoperative epistaxis $(\mathrm{n}=3)$, subcutaneous emphysema and displacement of the silicone tube. Conclusion:
Endonasal endoscopic dacryocystorhinostomy was shown to be a safe procedure, with a minimal incidence of postoperative complications. Its indication, however, is limited. Not all patients can undergo this technique without correction of nasal anatomy alterations. Though most surgeries with this technique present no difficulties, this procedure may be technically difficult, especially in the presence of hemorrhage or reduced nasal space.

Keywords: Dacryocystorhinostomy/methods; Lacrimal apparatus diseases; Lacrimal duct obstruction/surgery; Endoscopy/ methods; Retrospective studies

\section{REFERÊNCIAS}

1. Ben Simon GJ, Joseph J, Lee S, Schwarcz RM, McCann JD, Goldberg RA. External versus endoscopic dacryocystorhinostomy for acquired nasolacrimal duct obstruction in a tertiary referral center. Ophthalmology. 2005;112(8):1463-8.

2. Javate R, Pamintuan F. Endoscopic radiofrequency-assisted dacryocystorhinostomy with double stent: a personal experience. Orbit. 2005;24(1):15-22.

3. Onerci M, Orhan M, Ogretmenoglu O, Irkec M. Long-term results and reasons for failure of intranasal endoscopic dacryocystorhinostomy. Acta Otolaryngol. 2000;120(2):319-22

4. Gladstone GJ, Chen S, Nesi FA. Non-laser-assisted endoscopic dacryocystorhinostomy [video]. San Francisco: American Academy of Ophthalmology; 1994.

5. Knijnik D, Uebel VN, Santos RS. Dacriocistorrinostomia endoscópica endonasal em casos de epífora com vias lacrimais pérvias. Arq Bras Oftalmol. 2003;66(2):173-5.

6. Rebeiz EE, Shapshay SM, Bowlds JH, Pankratov MM. Anatomic guidelines for dacryocystorhinostomy. Laryngoscope. 1992;102(10):1181-4.

7. Woog JJ, Kennedy RH, Custer PL, Kaltreider SA, Meyer DR, Camara JG. Endonasal dacryocystorhinostomy: a report by the American Academy of Ophthalmology. Ophthalmology. 2001;108(12):2369-77.

8. Caldwell GW. Two new operations for obstruction of the nasal duct, with preservation of the canaliculi, and an incidental description of a new lacrymal probe. N York M J. 1893;57:581

9. Oguz H, San I, Verit A, Uzel I. Ophthalmic techniques described by Serefeddin Sabuncuoglu (1385-1468 AD). Clin Experiment Ophthalmol. 2004;32(2):192-5.

10. Bjork H. On intranasal dacryocystorhinostomy, with special reference to failures and their cause. Acta Otolaryngol. 1955;45(6):479-91.

11. Dolman PJ. Comparison of external dacryocystorhinostomy with nonlaser endonasal dacryocystorhinostomy. Ophthalmology. 2003;110(1):78-84.

12. Min YG, Jung HW, Kim CS. Prevalence study of nasal septal deformities in Korea: results of a nation-wide survey. Rhinology. 1995;33(2):61-5.

13. Oliver JM. The success rates for endonasal dacryocystorhinostomy. $\mathrm{Br} \mathrm{J}$ Ophthalmol. 2003;87(11):1431.

14. Fayet B, Racy E, Assouline M. Complications of standardized endonasal dacryocystorhinostomy with unciformectomy. Ophthalmology. 2004;111(4): $837-45$

15. Tsirbas A, Wormald PJ. Endonasal dacryocystorhinostomy with mucosal flaps Am J Ophthalmol. 2003;135(1):76-83.

16. Moura EM, Volpini M, Ianase M. Dacriocistorrinostomia transnasal vídeoendoscópica com Nd: YAG laser e diodo laser. Arq Bras Oftalmol. 2004; 67(5):807-14

17. Watkins LM, Janfaza P, Rubin PA. The evolution of endonasal dacryocystorhinostomy. Surv Ophthalmol. 2003;48(1):73-84. Review.

18. Blaylock WK, Moore CA, Linberg JV. Anterior ethmoid anatomy facilitates dacryocystorhinostomy. Arch Ophthalmol. 1990;108(12):1774-7.

19. Tsirbas A, Davis G, Wormald PJ. Mechanical endonasal dacryocystorhinostomy versus external dacryocystorhinostomy. Ophthal Plast Reconstr Surg. 2004; 20(1):50-6.

20. Sprekelsen MB, Barberan MT. Endoscopic dacryocystorhinostomy: surgical technique and results. Laryngoscope. 1996;106(2 Pt 1):187-9.

21. Hausler R, Caversaccio M. Microsurgical endonasal dacryocystorhinostomy with long-term insertion of bicanalicular silicone tubes. Arch Otolaryngol Head Neck Surg. 1998;124(2):188-91. 